\title{
THREE PROOFS OF MINKOWSKI'S SECOND INEQUALITY IN THE GEOMETRY OF NUMBERS
}

\author{
R. P. BAMBAH, ALAN WOODS and HANS ZASSENHAUS
}

(Received 28 December 1964)

\section{Dedicated to Kurt Mahler on his sixtieth birthday}

\section{Introduction}

Let $K$ be a bounded, open convex set in euclidean $n$-space $R_{n}$, symmetric in the origin 0 . Further let $L$ be a lattice in $R_{n}$ containing 0 and put

$$
m_{i}=\text { infimum } u_{i} \quad i=1,2, \cdots, n \text {; }
$$

extended over all positive real numbers $u_{i}$ for which $u_{i} K$ contains $i$ linearly independent points of $L$. Denote the Jordan content of $K$ by $V(K)$ and the determinant of $L$ by $d(L)$. Minkowski's second inequality in the geometry of numbers states that

$$
m_{1} m_{2} \cdots m_{n} V(K) \leqq 2^{n} d(L) .
$$

Minkowski's original proof has been simplified by Weyl [6] and Cassels [7] and a different proof has been given by Davenport [1].

Professor Mahler, during a seminar at Notre Dame University, suggested to the authors that it would be worthwhile to reexamine these proofs with a view to possible generalisations. Each author then gave a proof, one based on Weyl's paper [6] and two on Davenport's [1]. These three proofs are given here.

In Weyl's proof all considerations are made in the quotient space determined by the lattice $L$. The aim of the first proof is to show that the existence of the quotient space is needed only to deduce the so-called monotone property, thus suggesting that the theorem is true for some point sets other than lattices. Davenport's proof of (1) depends on certain functions constructed in the course of the argument being continuous. He states without proof that the construction can be made to ensure this. The third proof here shows how this may be done. Earlier Siegel in lectures at New York University gave without proof a method for making this construction. The second proof shows that by working with iterated integrals instead of 
Jordan contents Davenport's proof can be made independent of this continuity. Theorem A of this argument, a variant of the MinkowskiBlichfeldt theorem, may have some independent interest.

\section{Reduction of the problem}

The following normalisation is common to all three proofs. It is well known and easy to prove that there exist $n$ linearly independent points $F_{1}, F_{2}, \cdots, F_{n}$ of $L$ such that $F_{i}$ lies on the boundary of $m_{i} K$ for each $i=1,2, \cdots, n$. It is then possible to select a basis $P_{1}, P_{2}, \cdots, P_{n}$ of $L$ so that the linear space generated by the points $P_{1}, P_{2}, \cdots, P_{i}$ is the same as that generated by $F_{1}, F_{2}, \cdots, F_{i}$, and the intersection of this linear space with $L$ is the $i$-dimensional lattice $L_{i}$ generated by the points $P_{1}$, $P_{2}, \cdots, P_{i}$ for $i=1,2, \cdots, n$. Since the inequality (1) is invariant with respect to the full linear group of transformations of $R_{n}$ it follows that without loss of generality $L=L_{n}$ may be assumed to be the integral lattice and $P_{1}, P_{2}, \cdots, P_{n}$ the points given by

$$
P_{i}=\left(\delta_{i 1}, \delta_{i 2}, \cdots, \delta_{i n}\right) \quad i=1,2, \cdots, n ;
$$

where $\delta_{i j}$ is the Kronecker delta.

\section{First proof}

Let $C$ be a bounded Lebesgue measurable point set and $S$ a discrete point set in $R_{n}$. For $s \in S$ and a positive integer $m$ the set of points contained in $C+s$ and exactly $m-1$ of the sets $C+t, t \in S-s$, simultaneously is measurable with measure $M_{m}$ say. Let $k$ be the largest integer for which $M_{k}$ is not zero and put

$$
D(C, S, s)=M_{1}+\frac{1}{2} M_{2}+\frac{1}{3} M_{3}+\cdots+\frac{1}{k} M_{k} .
$$

The affine invariance of Lebesgue measure implies that

(A) If $x, y \in R_{n}$ then $D(C+x, S+y, s+y)=D(C, S, s)$;

(B) If $T$ is a nonsingular linear transformation of $R_{n}$ with determinant $|T|$ then

$$
D(T C, T S, T s)=\|T\| D(C, S, s) .
$$

For $i<n$ we convene that $R_{i}$ is the subspace of $R_{n}$ composed of those points for which the last $n-i$ coordinates vanish. Denote by $C\left[x_{i+1}, x_{i+2}, \cdots\right.$, $x_{n}$ ] the section of $C$ consisting of those points of $C$ whose last $n-i$ coordinates have the fixed values $x_{i+1}, x_{i+2}, \cdots, x_{n}$ respectively. Now if $S \subset R_{i}$ then for any point $t \in S$ the section of $C+t$ consisting of those points of $C+t$ whose 
last $n-i$ coordinates have the fixed values $x_{i+1}, x_{i+2}, \cdots, x_{n}$ respectively is given by $C\left[x_{i+1}, x_{i+2}, \cdots, x_{n}\right]+t$ and therefore by Fubini's theorem

(C) If $S \subset R_{i}$ then $D(C, S, s)=\int_{R_{n-i}} D\left(C\left[x_{i+1}, x_{i+2}, \cdots, x_{n}\right], S, s\right) d x_{i+1} \cdots d x_{n}$ where $D\left(C\left[x_{i+1}, \cdots, x_{n}\right], S, s\right)$ is understood to be the corresponding function in $R_{i}$.

By (A) it follows that $D\left(C, L_{i}, s\right)$ is independent of $s$ so in this case we write $D\left(C, L_{i}\right)$ in place of $D\left(C, L_{i}, s\right)$.

Denote by $\Pi_{i}$ the set of all points $\left(x_{1}, \cdots, x_{n}\right) \in R_{n}$ for which

$$
0 \leqq x_{j}<1 \quad j=1,2, \cdots, i ;
$$

Thus $\Pi_{1}$ is a fundamental region of $R_{n}$ modulo $L_{i}$. Let $C / L_{i}$ denote the set of all points of $C$ reduced modulo $L_{i}$ to $\Pi_{i}$ and $m\left(C / L_{i}\right)$ its measure. A fundamental principle of the geometry of numbers - the MinkowskiBlichfeldt theorem - may be stated as

$$
D\left(C, L_{i}\right)=m\left(C / L_{i}\right)
$$

Proof. $m$ points of $C$ are congruent modulo $L_{i}$ and congruent to no other points of $C$ if and only if each of the $m$ points lies in that part of $C$ which is covered by exactly $m-1$ of the sets $C+z, z \in L_{i}-0$. Since this part of $C$ has measure weighted by the factor $1 / m$ in $D\left(C, L_{i}\right)$ the result follows.

It follows immediately that

(E) If $C_{1}, C_{2}$ are bounded Lebesgue measurable subsets of $R_{n}$ such that $C_{1} \subset C_{2}$ then $D\left(C_{1}, L_{i}\right) \leqq D\left(C_{2}, L_{i}\right)$.

A subset $X$ of $R_{n}$ is said to be star with respect to a point $x \in X$ if whenever $y \in X$ so is the complete line segment joining $y$ to $x$ contained in $X$. The particular property of such sets required here is that if $t \geqq 1$ then

$$
X \subset t X+(x-t x)
$$

that is to say $t X$ may be translated into a position so as to cover $X$. We observe that a convex set is star with respect to any point in it. For a given $t \geqq 1$ denote by $T_{i}$ the linear transformation

$$
T_{i}\left(x_{1}, x_{2}, \cdots, x_{n}\right)=\left(t x_{1}, t x_{2}, \cdots, t x_{i}, x_{i+1}, \cdots, x_{n}\right)
$$

and by $K$ the convex body of the introduction. Then

$$
T_{i}\left(K\left[x_{i+1}, \cdots, x_{n}\right]\right)=\left(T_{i} K\right)\left[x_{i+1}, \cdots, x_{n}\right]
$$

and so $\left(T_{i} K\right)\left[x_{i+1}, \cdots, x_{n}\right]$ may be translated into a position in the same section so as to contain $K\left[x_{i+1}, \cdots, x_{n}\right]$. Hence (A) and (E) imply

$$
D\left(K\left[x_{i+1}, \cdots, x_{n}\right], L_{i}\right) \leqq D\left(\left(T_{i} K\right)\left[x_{i+1}, \cdots, x_{n}\right], L_{i}\right)
$$

and therefore also by (C)

$$
D\left(K, L_{i}\right) \leqq D\left(T_{i} K, L_{i}\right)
$$


LEMMA $1 . D\left(t K, L_{i}\right) \geqq t^{n-i} D\left(K, L_{i}\right)$.

Proof. If $T^{(n-i)}$ denotes the transformation

$$
T^{(n-i)}\left(x_{1}, \cdots, x_{n}\right)=\left(x_{1}, \cdots, x_{i}, t x_{i+1}, \cdots, t x_{n}\right)
$$

then $t K=T^{(n-i)} T_{i} K$ and, using (B) and $(\mathrm{F})$,

$$
D\left(t K, L_{i}\right)=D\left(T^{(n-i)} T_{i} K, T^{(n-i)} L_{i}\right)=\left\|T^{(n-i)}\right\| D\left(T_{i} K, L_{i}\right) \geqq t^{n-i} D\left(K, L_{i}\right) .
$$

LEMMa 2. If $K$ contains no point of $L_{n}-L_{i}$ then

$$
D\left(\frac{1}{2} K, L_{n}\right)=D\left(\frac{1}{2} K, L_{n-1}\right)=\cdots=D\left(\frac{1}{2} K, L_{i}\right) \text {. }
$$

PRoof. If $x \in L_{n}-L_{i}$ then $\frac{1}{2} K+x$ does not intersect $\frac{1}{2} K$ for otherwise the point $x$ is in $K$. The lemma then follows from the definition of $D\left(\frac{1}{2} K, L_{i}\right)$.

The inequality (1) now follows quickly, for by the definition of $m_{i+1}$ for $i=0,1, \cdots, n-1$ no point of $L_{n}-L_{i}$ lies in $m_{i+1} K$ whence by lemma 2 , $D\left(\frac{1}{2} m_{i+1} K, L_{i+1}\right)=D\left(\frac{1}{2} m_{i+1} K, L_{i}\right)$ and therefore also by lemma 1 ,

$$
\begin{aligned}
1 \geqq D\left(\frac{1}{2} m_{n} K, L_{n}\right) & =D\left(\frac{1}{2} m_{n} K, L_{n-1}\right) \geqq \frac{m_{n}}{m_{n-1}} D\left(\frac{1}{2} m_{n-1} K, L_{n-2}\right) \\
& \geqq \frac{m_{n}}{m_{n-1}}\left(\frac{m_{n-1}}{\bar{m}_{n-2}}\right)^{2} \cdots\left(\frac{m_{2}}{m_{1}}\right)^{n-1} D\left(\frac{1}{2} m_{1} K, L_{0}\right) \\
& =m_{n} m_{n-1} \cdots m_{1}\left(\frac{1}{2}\right)^{n} V(K) .
\end{aligned}
$$

\section{Second proof}

By the definition of $m_{i}$, it is clear that if $X$ is a point of $L_{n}$ in $m_{i} K$ then $x_{i}=x_{i+1}=\cdots=x_{n}=0$. By convexity and symmetry of $K$ the following three statements are equivalent:

(i) The sets $\frac{1}{2} m_{i} K, \frac{1}{2} m_{i} K+A$ overlap;

(ii) $\frac{1}{2} m_{i} K$ contains two points $X, Y$ such that $A=X-Y$;

(iii) $A$ lies in $m_{i} K$.

In particular if $\frac{1}{2} m_{i} K$ has two points $X, Y$ such that $A=X-Y$ lies in $L_{n}$ then $x_{i}=y_{i}, \cdots, x_{n}=y_{n}$.

In the rest of this proof we will assume that each function that appears in any integral is nonnegative and is nonzero only in some bounded set, not necessarily independent of the function. The symbol

$$
\int_{-\infty}^{\infty} \cdots \int_{-\infty}^{\infty} f\left(x_{n}, \cdots, x_{1}\right) d x_{1} d x_{2} \cdots d x_{i}
$$

will stand for the iterated Riemann integral

$$
\int_{-\infty}^{\infty} d x_{i} \int_{-\infty}^{\infty} d x_{i-1} \cdots \int_{-\infty}^{\infty} f\left(x_{n}, \cdots, x_{1}\right) d x_{1} .
$$


We next prove a few almost obvious results about these integrals.

Lemma 1. Suppose that $I=\int_{-\infty}^{\infty} \cdots \int_{-\infty}^{\infty} f\left(x_{n}, \cdots, x_{1}\right) d x_{1} \cdots d x_{i}$ $1 \leqq i \leqq n$ exists. Then, given real numbers $a_{1}, \cdots, a_{i}$,

$$
I=\int_{-\infty}^{\infty} \cdots \int_{-\infty}^{\infty} f\left(x_{n}, \cdots, x_{i+1}, x_{i}-a_{i}, \cdots, x_{1}-a_{1}\right) d x_{1} \cdots d x_{i} .
$$

Proof. For $i=1$ it follows from the definition and by induction for $i>1$.

LEMma 2. Suppose that $I=\int_{-\infty}^{\infty} \cdots \int_{-\infty}^{\infty} f\left(x_{n}, \cdots, x_{1}\right) d x_{1} \cdots d x_{n}$ exists. Then for given numbers $a_{1}>0, \cdots, a_{i}>0$ the integral $I(a)=\int_{-\infty}^{\infty} \cdots$ $\int_{-\infty}^{\infty} f\left(x_{n}, \cdots, x_{i+1},\left(x_{i} / a_{i}\right), \cdots,\left(x_{1} / a_{1}\right)\right) d x_{1} \cdots d x_{i}$ exists and $I(a)=a_{1} \cdots a_{i} I$.

Proof. For $i=1$ by definition and for $i>1$ by induction.

LEMMA 3. (i) If

and

$$
I_{1}=\int_{-\infty}^{\infty} \cdots \int_{-\infty}^{\infty} f\left(x_{n}, \cdots, x_{1}\right) d x_{1} \cdots d x_{n}
$$

$$
I_{2}=\int_{-\infty}^{\infty} \cdots \int_{-\infty}^{\infty} g\left(x_{n}, \cdots, x_{1}\right) d x_{1} \cdots d x_{n}
$$

both exist then so does

$$
I=\int_{-\infty}^{\infty} \cdots \int_{-\infty}^{\infty}(f+g) d x_{1} \cdots d x_{n}
$$

and $I=I_{1}+I_{2}$.

(ii) If in (i), $f \leqq g$ for all points $X$ then $I_{1} \leqq I_{2}$.

Proof. Clear.

TheOREM A. Suppose that $S$ is a bounded set in $R_{n}$. Let $\chi(X)=$ $\chi\left(x_{n}, \cdots, x_{1}\right)$ be its characteristic function. Suppose further that

$$
I=\int_{-\infty}^{\infty} \cdots \int_{-\infty}^{\infty} \chi(X) d x_{1} \cdots d x_{n} \text { exists and } I>1 .
$$

Then the sets $S+A, A \in L$ overlap so that there exist points $X, Y$ in $S$, $X \neq Y$, such that $X-Y=A \in L$.

Proof. Suppose $S$ lies in the box $\left|x_{i}\right| \leqq k$. Suppose further that the sets $S+A, A \in L$ do not overlap. For a fixed positive integer $N$ consider the set $\Sigma$ of points $A$ of $L$ such that $1 \leqq a_{i} \leqq N, i=1, \cdots, n$. Define

$$
F(X)=\sum_{\Lambda \in \Sigma} \chi(X-A)
$$

Since the sets $S+A$ do not overlap $F(X) \leqq 1$ for all $X$. Since the sets lie in the box $B$ given by $-k \leqq x_{i} \leqq N+k$, so $F(X)=0$ if $X \notin B$. By the lemmas 1 and 3 , 
so that

$$
\begin{aligned}
(N+2 k)^{n} & \geqq \int_{-\infty}^{\infty} \cdots \int_{-\infty}^{\infty} F(X) d x_{1} \cdots d x_{n} \\
& =\sum_{A \in \Sigma} \int_{-\infty}^{\infty} \cdots \int_{-\infty}^{\infty} \chi(X-A) d x_{1} \cdots d x_{n} \\
& =\sum_{A \in \Sigma} \int_{-\infty}^{\infty} \cdots \int_{-\infty}^{\infty} \chi(X) d x_{1} \cdots d x_{n} \\
& =(N+1)^{k} I
\end{aligned}
$$

$$
I \leqq\left(\frac{N+2 k}{N+1}\right)^{n}
$$

By making $N \rightarrow \infty$, we get $I \leqq 1$ and the theorem follows.

LEMMA 4. It is possible to construct sets $K_{1}, \cdots, K_{n}$ such that

(1) $K_{1}=\frac{1}{2} m_{1} K$,

(2) $K_{i} \subset \frac{1}{2} m_{i} K$

(3) If $X, Y \in K_{i}$

for $i>1$ and $x_{i}=y_{i}, \cdots, x_{n}=y_{n}$ then there exist points $X^{\prime}, Y^{\prime}$ in $K_{i-1}$ such that $X-Y=X^{\prime}-Y^{\prime}$ and

(4) If $\chi_{i}(X)=\chi_{i}\left(x_{n}, \cdots, x_{1}\right)$ is the characteristic function of $K_{i}$ then

exists and

$$
V_{i}=\int_{-\infty}^{\infty} \cdots \int_{-\infty}^{\infty} x_{i}\left(x_{n}, \cdots, x_{1}\right) d x_{1} \cdots d x_{n}
$$

$$
V_{i}= \begin{cases}\left(\frac{1}{2} m_{1}\right)^{n} V(K) & \text { if } i=1 \\ \left(\frac{m_{i}}{m_{i-1}}\right)^{n-i+1} V_{i-1} & \text { if } i>1\end{cases}
$$

Proof. Take $K_{1}=\frac{1}{2} m_{1} K$. To prove (4) we observe that $K_{1}$ has volume $\left(\frac{1}{2} m_{1}\right)^{n} V(K)$ and since $K_{1}$ is convex all its sections by hyperplanes of various dimensions are convex and hence have volumes in their appropriate dimensions. Therefore

$$
\begin{aligned}
\left(\frac{1}{2} m_{1}\right)^{n} V(K) & =V\left(K_{1}\right)=\int_{-\infty}^{\infty} \cdots \int_{-\infty}^{\infty} \chi(X) d x_{1} \cdots d x_{n} \\
& =\int_{-\infty}^{\infty} d x_{n} \int_{-\infty}^{\infty} \cdots \int_{-\infty}^{\infty} \chi(X) d x_{1} \cdots d x_{n-1}=\cdots \\
& =\int_{-\infty}^{\infty} d x_{n} \int_{-\infty}^{\infty} d x_{n-1} \cdots \int_{-\infty}^{\infty} \chi(X) d x_{1}=V_{1}
\end{aligned}
$$

so that $K_{1}$ has the required properties.

Suppose that $K_{1}, \cdots, K_{i-1}(2 \leqq i \leqq n)$ have been constructed to satisfy (1) through (4). For each point $\left(0,0, \cdots, 0, x_{i}, \cdots, x_{n}\right)$ of the projection of $\frac{1}{2} m_{i-1} K$ on $x_{1}=\cdots=x_{i-1}=0$ choose a point 


$$
\left(\varphi_{1}\left(x_{i}, \cdots, x_{n}\right), \cdots, \varphi_{i-1}\left(x_{i}, \cdots, x_{n}\right), x_{i}, \cdots, x_{n}\right) \text { of } \frac{1}{2} m_{i-1} K .
$$

Define $K_{i}$ to be the set of points $Y$ where

$$
\begin{aligned}
y_{1} & =x_{1}+\left(\frac{m_{i}}{m_{i-1}}-1\right) \varphi_{1}\left(x_{i}, \cdots, x_{n}\right) \\
y_{2} & =x_{2}+\left(\frac{m_{i}}{m_{i-1}}-1\right) \varphi_{2}\left(x_{i}, \cdots, x_{n}\right) \\
& \ldots \\
y_{i-1} & =x_{i-1}+\left(\frac{m_{i}}{m_{i-1}}-1\right) \varphi_{i-1}\left(x_{i}, \cdots, x_{n}\right) \\
y_{i} & =\frac{m_{i}}{m_{i-1}} x_{i} \\
& \cdots \\
y_{n} & =\frac{m_{i}}{m_{i-1}} x_{n}
\end{aligned}
$$

and $X \in K_{i-1}$.

Since $X=\left(x_{1}, \cdots, x_{n}\right)$ and $\tilde{X}=\left(\varphi_{1}, \cdots, \varphi_{i-1}, x_{i}, \cdots, x_{n}\right)$ lie in $\frac{1}{2} m_{i-1} K$ which is convex so does $Z=\left\{X+\left(m_{i-1} / m_{i}-1\right) X\right\} \mid m_{i-1} / m_{i}$. Therefore $Y=\left(m_{i} / m_{i-1}\right) Z$ lies in $\frac{1}{2} m_{i} K$. This proves (1) and (2) for $K_{i}$ while (3) is a direct consequence of the definition.

$$
\begin{aligned}
V_{i}= & \int_{-\infty}^{\infty} \cdots \int_{-\infty}^{\infty} x_{i}\left(y_{n}, \cdots, y_{1}\right) d y_{1} \cdots d y_{n} \\
= & \int_{-\infty}^{\infty} \cdots \int_{-\infty}^{\infty} x_{i-1}\left(a^{-1} y_{n}, \cdots, a^{-1} y_{i}, y_{i-1}-(a-1) \varphi_{i-1}\left(a^{-1} y_{i}, \cdots, a^{-1} y_{n}\right)\right. \\
& \left.\cdots, y_{1}-(a-1) \varphi_{1}\left(a^{-1} y_{i}, \cdots, a^{-1} y_{n}\right)\right) d y_{1} \cdots d y_{n}
\end{aligned}
$$

where $a=\left(m_{i} / m_{i-1}\right)$, exists because if we write

$$
\chi\left(x_{n}, \cdots, x_{i}\right)=\int_{-\infty}^{\infty} \cdots \int_{-\infty}^{\infty} x_{i-1}\left(x_{n}, \cdots, x_{i}, x_{i-1}, \cdots, x_{1}\right) d x_{1} \cdots d x_{i-1}
$$

then $\chi$ exists by the induction hypothesis and equals

$$
\begin{aligned}
\int_{-\infty}^{\infty} \cdots \int_{-\infty}^{\infty} x_{i-1}\left(x_{n}, \cdots,\right. & x_{i}, x_{i-1}-(a-1) \varphi_{i-1}\left(a^{-1} x_{i}, \cdots, a^{-1} x_{n}\right), \cdots, \\
& \left.x_{1}-(a-1) \varphi_{1}\left(a^{-1} x_{i}, \cdots, a^{-1} x_{n}\right)\right) d x_{1} \cdots d x_{i-1}
\end{aligned}
$$

by lemma 1 , so that by lemma 2 ,

$$
\begin{aligned}
V_{i} & =\int_{-\infty}^{\infty} \cdots \int_{-\infty}^{\infty} \chi_{i-1}\left(a^{-1} y_{n}, \cdots, a^{-1} y_{i}, y_{i-1}, \cdots, y_{1}\right) d y_{1} \cdots d y_{n} \\
& =a^{n-i+1} \int_{-\infty}^{\infty} \cdots \int_{-\infty}^{\infty} x_{i-1}\left(x_{n}, \cdots, x_{i}, \cdots, x_{1}\right) d x_{1} \cdots d x_{n} \\
& =a^{n-i+1} V_{i-1} .
\end{aligned}
$$


This proves the lemma.

Proof of Minkowski's inequality (1). Suppose that $m_{1} \cdots m_{n} V(K)$ $>2^{n}$. Then by lemma 4

$$
V_{n}=m_{1} \cdots m_{n} 2^{-n} V(K)>1,
$$

and by theorem $A$ there exist points $X, Y$ in $K_{n}$ such that $0 \neq X-Y \in L_{n}$. Since $K_{n} \subset \frac{1}{2} m_{n} K$ this implies that $x_{n}=y_{n}$ and by property (1) of the sets $K_{i}, K_{n-1}$ contains points $X^{(1)}, Y^{(1)}$ such that

$$
X-Y=X^{(1)}-Y^{(1)} \text {. }
$$

Since $K_{n-1} \subset \frac{1}{2} m_{n-1} K$ this implies that $x_{n-1}^{(1)}=y_{n-1}^{(1)}, x_{n}^{(1)}=y_{n}^{(1)}$ and there exist points $X^{(2)}, Y^{(2)}$ in $K_{n-2}$ with

$$
X-Y=X^{(2)}-Y^{(2)} \text {. }
$$

Repeating this argument a number of times we obtain points $X^{*}, Y^{*}$ in $\frac{1}{2} m_{1} K$ such that $X-Y=X^{*}-Y^{*}$. But $X^{*}-Y^{*} \in L_{n}$ implies that $X^{*}=Y^{*}$ and $X-Y=0$, which is a contradiction. This proves the theorem.

\section{Third proof}

In view of the fact that the Jordan content of $K$ and the successive minima $m_{1}, \cdots, m_{n}$ are continuous functions of $K$ it is only necessary to prove the inequality for strictly convex bodies $K$. Thus it is assumed that from now on $K$ is strictly convex.

As with Davenport (1) this proof depends upon the inductive construction of sets $K_{1}, \cdots, K_{n}$ so that $K_{i}$ has the following properties

(1) $K_{i}$ is a bounded Jordan measurable open star body symmetric in the origin;

(2) $K_{i} \subset \frac{1}{2} m_{i} K$

(3) $K_{i} \cap R_{j} \subset \frac{1}{2} m_{j} K$ for $j=1,2, \cdots, i-1$;

(4) $V\left(K_{i}\right)=\left(\frac{m_{i}}{m_{i-1}}\right)^{n-i+1} V\left(K_{i-1}\right)$ if $i \geqq 2$;

(5) $V\left(K_{1}\right)=\left(\frac{1}{2}\right)^{n} m_{1}^{n} V(K)$.

(6) No two translates of $K_{i}$ by points of $L_{n}$ have a point in common.

Putting $K_{1}=\frac{1}{2} m_{1} K$ properties (1)-(6) are satisfied for $i=1$. Assuming that $K_{i}$ has been constructed to satisfy $(1)-(6)$ define $K_{i+1}$ as follows.

Let $X \in R_{n}$. Since $K$ is strictly convex the linear manifold $X+R_{i}$ contains a unique point, $f(X)$ say, that is closest to the origin in the metric determined by $K$. If the coordinates of $X$ are $x_{1}, \cdots, x_{n}$ then the coordinates 
of $f(X)$ are independent of $x_{1}, \cdots, x_{i}$ and continuous functions of $x_{i+1}$, $\cdots, x_{n}$. Moreover as $K$ is star and symmetric in the origin so

$$
f(t X)=t f(X) \text { for all real } t .
$$

Any point of $R_{n}$ can now be written in the form

$$
A+f(X)
$$

where $A \in R_{i}$ and $A, f(X)$ are uniquely determined. Define $K_{i+1}$ to be the set of all points

$$
A+\left(\frac{m_{i+1}}{m_{i}}\right) f(X)
$$

for which $A+f(X) \in K_{i}$. It remains to verify that $K_{i+1}$ has properties (1) $-(6)$.

Let $P \in K_{i+1}$ so that $P=A+\left(m_{i+1} / m_{i}\right) f(X)$ where $A+f(X) \in K_{i}$. Since $K_{i}$ is star and symmetric in the origin it follows that $t A+t f(X) \in K_{i}$ for $|t| \leqq 1$. Therefore also

$$
t A+\left(\frac{m_{i+1}}{m_{i}}\right) t f(X)=t P \in K_{i+1}
$$

and $K_{i+1}$ is star and symmetric the origin. As $f(X)$ is continuous in each of its coordinates so $K_{i+1}$ is open and Jordan measurable and so satisfies (1). Also $A+f(X) \in K_{i} \subset \frac{1}{2} m_{i} K$ and therefore by construction $f(X) \in \frac{1}{2} m_{i} K$. By the convexity of $\frac{1}{2} m_{i} K$ it follows that

i.e.

$$
\left(\frac{m_{i}}{m_{i+1}}\right)(A+f(X))+\left(1-\frac{m_{i}}{m_{i+1}}\right) f(X) \in \frac{1}{2} m_{i} K
$$

hence

$$
\left(\frac{m_{i}}{m_{i+1}}\right) A+f(X) \in \frac{1}{2} m_{i} K
$$

and $K_{i}$ satisfies (2).

$$
A+\left(\frac{m_{i+1}}{m_{i}}\right) f(X) \in \frac{1}{2} m_{i+1} K
$$

By construction $K_{i+1} \cap R_{j}=K_{i} \cap R_{j}$ for $j=1,2, \cdots, i$ so that $K_{i+1}$ satisfies (3).

By Fubini's theorem $K_{i+1}$ has the same Jordan content as the point set obtained from $K_{i}$ by dilating the last $n-i$ coordinates by the factor $m_{i+1} / m_{i}$. Hence

and $K_{i+1}$ has property (4).

$$
V\left(K_{i+1}\right)=\left(\frac{m_{i+1}}{m_{i}}\right)^{n-i} V\left(K_{i}\right)
$$


Property (5) refers only to the case $i=1$ so it remains to verify (6). If two translates of $K_{i+1}$ by points of $L_{n}$ have a point in common then there exist a pair of points $X, Y$ say in $K_{i+1}$ such that $X-Y \in K_{i+1}$. Since $K_{i+1} \subset \frac{1}{2} m_{i+1} K$ it follows that $X-Y \in L_{i} \subset R_{i}$. Now

$$
X=A+\left(\frac{m_{i+1}}{m_{i}}\right) f(Z), \quad Y=B+\left(\frac{m_{i+1}}{m_{i}}\right) f\left(Z^{\prime}\right)
$$

where $A, B \in R_{i}$ and

$$
A+f(Z) \in K_{i}, \quad B+f\left(Z^{\prime}\right) \in K_{i} .
$$

As $X-Y \in R_{i}$ so $f(Z)=f\left(Z^{\prime}\right)$ and

$X-Y=A-B=(A+f(Z))-\left(B+f\left(Z^{\prime}\right)\right)$. Thus there are two distinct points of $K_{i}$ whose difference is in $L_{n}$ and therefore two translates of $K_{i}$ by $L_{n}$ have a point in common which is impossible. $K_{i+1}$ therefore has properties (1)-(6).

The sets $K_{1}, \cdots, K_{n}$ having been constructed property (6) implies that

$$
V\left(K_{n}\right) \leqq 1
$$

or $m_{1} m_{2} \cdots m_{n} V(K) \leqq 2^{n}$ which completes the proof.

The above construction of $K_{n}$ raises the question whether a convex centrally symmetric body exists with properties similar to $(1)-(6)$. Further if $C_{i}$ denotes the set of points in $R_{i}$ that are a distance $m_{i}$ from the origin in the metric determined by $K$ and if $D$ denotes the set of all points that are closer to the origin than to any point of $\bigcup_{i=1}^{n} C_{i}$ then it can be shown that $D$ is an open star body symmetric in the origin the translates of which by points of $L_{n}$ do not intersect. This raises the question of whether the content of $D$ is large enough, i.e. at least as large as $V\left(K_{n}\right)$, to produce another proof of the inequality.

\section{References}

[1] H. Davenport, Minkowski's inequality for the minimum associated with a convex body, Quart. J. Math. Oxford 10 (1939), 119-121.

[2] K. Mahler, Über die Annäherung algebraischer Zahlen durch periodische Algorithmen, Acta Mathematica 68 (1937), $109-144$.

[3] H. Minkowski, Geometrie der Zahlen, Teubner 1896, Chapter 5.

[4] C. L. Siegel, Geometry of Numbers, Lectures at New York University.

[5] H. Zassenhaus, Modern developments in the Geometry of Numbers, Bull. Am. Math. Soc. 67 (1961), 427-439.

[6] H. Weyl, On geometry of numbers. Proc. Lond. Math. Soc. (2) 47 (1939-40), 268-289.

[7] J. W. S. Cassels, An Introduction to the geometry of numbers, Springer, 1959.

The Ohio State University

Columbus, Ohio 